\title{
Codependencia y psicoterapia interpersonal
}

RESUMEN: La codependencia hace referencia a una actitud obsesiva y compulsiva hacia el control de otras personas y las relaciones, fruto de la propia inseguridad.

El abordaje de la codependencia desde la psicoterapia interpersonal se basa en un enfoque pluralista, no doctrinario y empírico, que tiene características específicas. Su peculiaridad se encuentra en sus estrategias, no en sus técnicas. En ella se abordan cuatro áreas: duelo, disputas personales, déficits interpersonales y transiciones de rol. PALABRAS CLAVE: vínculo, codependencia, psicoterapia interpersonal.

\begin{abstract}
The codependency refers to an obsessive and compulsive attitude towards the control of other persons and their relations, due to his or her own insecurity.

The approach to the codependency through the interpersonal psychotherapy is based on a pluralist, but not doctrinary or empiric view, which presents especific characteristics. Its singularity appears not through its tecnics but in its stategies, in which we study for areas: grief, personal arguments, social skills deficits and role transitions.
\end{abstract}

KEY WORDS: attachment, codependency, interpersonal psychotherapy.

\section{Introducción.-}

El concepto de codependencia se comenzó a utilizar en los años setenta para describir a la persona, sea ésta familiar o amigo, que tiene una relación directa e íntima con un alcohólico y que le facilita continuar con la adicción. Más tarde se ha hecho referencia al codependiente como aquella persona que se dedica a cuidar, corregir y salvar a un drogodependiente, involucrándose en sus situaciones de vida conflictivas, sufriendo y frustrándose ante sus repetidas recaídas, llegando a adquirir características y conductas tan erróneas como las del propio adicto (1). Posteriormente se ha ido generalizando a familiares de personas con otras dependencias, con enfermedad crónica (esclerosis múltiple, insuficiencia renal, trastornos alimentarios, cáncer, esquizofrenia, enfermedad de Alzheimer) e incluso a profesionales de la salud (2).

Pero la codependencia ha sido definida en múltiples sentidos: como un esquema de vida disfuncional que emerge en la familia de origen produciendo un estancamiento en el desarrollo y siendo su resultado una hiperreacción del codependiente a lo externo a él y una hiporreacción o baja sensibilidad a lo interno a él (3); como la conducta de una persona esencialmente normal que realiza un esfuerzo para ajustarse a un cónyuge y a un acontecimiento vital estresante (4); como un patrón de dolorosa dependencia de otros, con comportamientos compulsivos y de búsqueda de la aprobación para intentar encontrar 
seguridad, autoestima e identidad (5) (6); como un patrón de rasgos de personalidad claramente identificables que presentan los integrantes de una familia que tiene un miembro afectado por una adicción a sustancias químicas (7); como una enfermedad primordial presente en cada miembro de la familia adicta, que es a menudo peor que la propia enfermedad, y que tiene sus propias manifestaciones psicosomáticas (8); como una característica emocional y psicológica de la conducta que aparece como consecuencia de un conjunto de reglas opresoras que impiden la demostración abierta de sentimientos y el diálogo sobre problemas personales e interpersonales (9). También se ha señalado a la codependencia como una patología del vínculo que se manifiesta por la excesiva tendencia a encargarse o a asumir las responsabilidades de otros (10), o como un defecto en la realización de dos de los cometidos más importantes de la niñez: la autonomía y la identidad (11). Incluso el concepto de codependencia se ha utilizado para describir un patrón exagerado de dependencia que hace llegar al individuo hasta la negligencia de sí mismo y debilita su propia identidad (12). Y también se ha indicado con este término a cuando algún familiar se involucra de forma obsesiva en los problemas de un adicto hasta el punto de vivir por y para él y desequilibrando su propia vida en las áreas personal, familiar, laboral y social. De modo que el codependiente pierde el control de su propia vida y de sus límites, invirtiendo toda su energía en el adicto con necesidad de pertenecer y ser útil (13).

Sin embargo, se ha criticado la tendencia a conceptualizar los comportamientos interpersonales como una dependencia (14). Y se ha visto el concepto de codependencia como una exageración ya que se ha considerado que la mujer del alcohólico se encuentra bajo presión psicológica y no sorprende que tenga problemas de ansiedad, depresión y baja autoestima (15). Parece así mismo excesivo considerar como patológicos comportamientos asociados a cualidades de la mujer (cuidar y proteger a los demás) y no reconocer el comportamiento de las esposas como una respuesta de una persona en buen estado con relación a otra en peor estado (16). Por eso se ha afirmado, que aunque los hombres pueden ser en teoría codependientes, se ha hecho principalmente referencia a una patología de la mujer (15). Aunque en principio la codependencia no es un fenómeno específico atribuible a las mujeres, puede que los condicionamientos sociales y culturales hayan determinado una alta prevalencia de este problema en ellas (17).

También se ha señalado que los codependientes tienen los mismos rasgos que los hijos adultos de alcohólicos: exigencia de controlar a los otros y su ambiente, y un temor a la asertividad junto a una demanda extrema de amor y aprobación (18).

Se ha llegado a ver a la codependencia como la otra cara de la adicción (19), o como una adicción de orden afectivo (20), o como una relación adictiva a una persona y sus problemas (21), porque esta relación puede ser tan compulsiva e impulsiva como una adicción (22).

Por tanto, la codependencia ha sido explicada desde tres puntos de vista:

a) Como una enfermedad primaria de un sistema familiar disfuncional y que una vez desencadenada seguirá su curso y afectará a uno o más miembros de la familia (23). 
b) Como un trastorno de personalidad previo de uno o más miembros de la familia en interacción con la conducta del adicto que facilitan la adicción, la encubren y la mantienen (24).

c) Y como la conducta de una persona esencialmente normal que realiza un esfuerzo para ajustarse a un cónyuge y a un acontecimiento vital estresante (4).

Aunque se ha señalado que hay dependencias relacionales con entidad propia como las denominadas dependencias emocionales como la adicción al amor (25), interdependencia, dependencia afectiva y otras secundarias a trastornos adictivos (sobre todo a drogas y alcohol) como la codependencia y la bidependencia (doble dependencia a sustancias y afectiva) (26).

Parecería, más bien, que la codependencia pudiera ser una entidad nosológica que hace referencia a un tipo de dependencia emocional y con un vínculo patológico similar al que caracteriza a aquellos sujetos que mantienen otro tipo de adicción. La diferencia entre el adicto y el codependiente no se debe a la dinámica subyacente a la adicción sino al objeto de consumo (19).

En todo caso, la codependencia es una adicción a una persona y a sus problemas (23), y se ha llegado a afirmar que la adicción y la codependencia son la misma enfermedad porque comparten las mismas características: negación, obsesión, compulsión y pérdida de control (27).

La codependencia sería, pues, una forma de adicción como la drogodependencia, la ludopatía u otras adicciones.

El término hoy día hace referencia a la actitud obsesiva y compulsiva hacia el control de otras personas y las relaciones, fruto de la propia inseguridad. Y es condición necesaria que el trastorno o la enfermedad de la otra persona sea crónica (28), pero llevando asociada la esperanza de que puede ser curada.

La expresión sintomática del codependiente se caracteriza por la necesidad de tener el control sobre el otro, por una baja autoestima, por un autoconcepto negativo, por la dificultad para poner límites, por la represión de sus emociones, por hacer propios los problemas del otro, por la negación del problema, por ideas obsesivas y conductas compulsivas, por el miedo a ser abandonado, a la soledad o al rechazo, por su extremismo (o son hiperrresponsables o demasiado irresponsables). Además se siente víctima porque sacrifica su propia felicidad, tiene dificultad para la diversión y se juzga sin misericordia (29).

\section{Tipología de la codependencia (7)}

Se han identificado cuatro tipos de codependientes:

El codependiente directo que presenta uno de los comportamientos que genera más dificultades en el proceso terapéutico porque su conducta va desde proporcionarle la droga hasta dinero o el lugar donde pueda consumir la droga.

El codependiente indirecto mantiene una conducta de oposición declarada y objetiva a la adicción del familiar pero, a la vez, protege al adicto, y evitan que se responsabilice de sus acciones. 
El codependiente tolerante desempeña el rol de sufridor. Su rol no es modificar el comportamiento del adicto sino contemplar como se autodestruye pero queriendo sacar lo que queda de bueno y noble en él.

El codependiente perseguidor es el familiar más comprometido en controlar la conducta autodestructiva del adicto. Despliega un sistema de conducta para descubrirlo. Es el que opera con un control externo.

La codependencia puede también ser percibida en el comportamiento de los hijos de drogodependientes, de enfermos mentales graves y en los hijos que han perdido a un progenitor a una edad temprana. Cuatro suelen ser los roles de estos hijos (7) (30) (31).

El héroe de la familia o niño adulto es el hijo parentificado que adopta el rol de padre/ madre frente a sus hermanos, llegando a convertirse en el cuidador del progenitor drogodependiente, no juega ni fantasea como los demás niños ni disfruta de su niñez, asume las funciones importantes de la familia para que ésta no se desintegre. Y es utilizado por la familia para demostrarse a sí misma y a los demás que el sistema familiar funciona bien.

El niño perdido o silente con frecuencia es el segundo de la fratría, es el que pasa desapercibido en la constelación familiar, suele estar solo, tiene tendencia a la fantasía como válvula de escape de los problemas familiares, busca relaciones íntimas fuera de la familia y desarrolla una gran capacidad para mantenerse lejos física y psíquicamente.

El hijo problema o hijo crisis o cabeza de turco sirve de pararrayos para las tensiones que se crean dentro de la familia, manifiesta una conducta desafiante, crea conflictos, presenta agresividad no controlada y frecuenta ambientes de marginación social. Su modo de actuar es provocando problemas para proteger al padre contra una confrontación, proteger a la madre de poner al padre en una difícil posición, proteger a los hermanos centrando la ira del padre sobre sí mismo, y protegerse de sí mismo contra sensaciones de pasividad.

El hijo broma o mascota suele ser el menor de la fratría, es frágil e inmaduro, tiene muchos amigos, es simpático, su único objetivo es agradar a los demás, tiene siempre la intervención graciosa y oportuna, por lo que se le suele etiquetar de payaso.

\section{Desarrollo de la codependencia}

El término vinculación hace referencia a una relación objetal específica y supone una estructura neurofisiológica y la tendencia a buscar la relación con otro (32).

La madre, durante el embarazo, se prepara emocionalmente para recibir a su hijo, desarrollando la preocupación materna primaria que la convierte en la persona más indicada para establecer la interacción (33).

Esta relación se produce incluso antes del parto y adquiere una calidad que depende de la madre y del hijo, de sus dotaciones específicas y de la modulación y conjugación de ambos (34). 
El recién nacido utiliza los diferentes estados: de vigilancia, de excitación, de actividad motriz y de calidad afectiva (35) para controlar las tensiones endógenas o exógenas y organizar sus vivencias. Gran parte de los cuidados que le da la madre consisten en modular su estado, proporcionándole estimulaciones o protegiéndole contra dosis excesivas (36) (37).

El lactante percibe la intensidad, el ritmo, la modulación de la voz, las posturas y el tono muscular, y responde de una manera específica con la sonrisa, las vocalizaciones y los movimientos coordinados de los ojos y de la cabeza. Este es el modo de comunicación (38). De manera que madre e hijo se sumergen en la llamada fase fusional de Winnicot. Esto ocurre cuando la madre hace una identificación proyectiva con las necesidades del niño, lo que le permite realizar satisfactoriamente todas las tareas del proceso de maternización, es decir, la función materna es lo suficientemente buena (39).

El niño nace con la tendencia al establecimiento del vínculo porque posee una serie de capacidades sensoriales y se encuentra con una madre o sustituta especialmente predispuesta para este proceso.

El fallo en el vínculo podría dar como resultado un trastorno de relación.

Con la combinación de las dimensiones de afecto y control se establecieron cuatro tipos de vínculos (40) que equivalen a otros tantos de Bowlby (32). El vínculo I (equilibrio de afecto y protección) equivale al vínculo óptimo o apego normal de Bowlby. El vínculo II (afecto constrictivo) equivale al vínculo dependiente. El vínculo III (afecto deficitario o ausente) equivale al desapego o ausencia vincular. Y el vínculo IV (control sin afecto) equivale al vínculo ansioso. Entre los codependientes pueden encontrarse los vínculos II, III y IV, aunque quizás el más frecuente sea el vínculo IV (vinculación de poco afecto y sobreprotección excesiva).

Los cambios que ocurren en la interacción entre le niño y la figura de apego puede determinar la aparición del conflicto del vínculo. Este puede surgir cuando al niño se le comienza a exigir un comportamiento más adecuado a lo que se considera socialmente es deseable, instándole a adquirir mayor autonomía e independencia, cuando al mismo tiempo no tiene con la figura de apego una relación íntima, cálida y continua, en la que los dos encuentren alegría y satisfacción (41). El niño siente que si se preocupa y le presta servicio a la figura de apego es correspondido y si no se preocupa y no le sirve puede ser abandonado o rechazado.

El niño percibe el desinterés o desapego de la madre o figura sustituta. Y busca apoyo y compañía sometiéndose, halagándola y satisfaciéndola por todos sus medios. El niño permanece atento a sus señales que le informan sobre los gustos de la madre, y por supuesto evita cualquier agresión. Lo que genera que el niño sienta una particular debilidad centrada en la necesidad de protección que le hace buscar la compañía de la madre.

También la vinculación defectuosa puede generar lo que ha venido en denominarse la "absorción emocional" que tiene lugar cuando al niño no se le permite separarse de la madre en el momento oportuno. Si una madre o figura sustituta es sobreprotectora y no acaba de distanciarse y de aflojar los lazos para permitir que el niño se convierta en una persona independiente, entonces el niño no se vinculará adecuadamente (11). 
De manera que la codependencia puede forjarse a partir de las necesidades no satisfechas en el ser humano durante su infancia, las cuales han impedido una maduración conveniente para poder adaptarse a situaciones de relaciones interpersonales.

Cuando las necesidades físicas y emocionales del niño no son satisfechas de una manera adecuada, su self verdadero, auténtico va construyendo las etapas evolutivas con el apoyo de un yo subordinado que desarrolla roles que le permiten superar las experiencias problemáticas de la infancia, y que para sobrevivir le incitan a aprender a “servir a los demás”, descuidándose a sí mismo.

Los codependientes insisten en repetir las mismas conductas ineficaces que utilizaron cuando eran niños para sentirse aceptados, queridos o importantes y mediante esas conductas, buscan aliviar el dolor y la pena por sentirse abandonados. Sin embargo, paradójicamente las conductas codependientes perpetúan esos sentimientos.

Este vínculo defectuoso que establece el codependiente está colocado en la preocupación por lo que el otro piensa, en el miedo a la pérdida de la relación, sintiendo y vivenciando la culpa y siempre tratando de reparar. El codependiente percibe un mundo interrelacional peligroso, entre la necesidad de proteger y el temor a ser abandonado, anticipando la excesiva separación y sobre todo la pérdida de amor.

A pesar de la fragilidad del término codependencia se podría hipotetizar un modelo conceptual y comprensivo. Para la presencia de la codependencia se precisan unos factores predisponentes, precipitantes y de mantenimiento. El factor predisponente sería el fallo en el vínculo madre-hijo que no ha posibilitado que a través de la relación con la madre el niño haya podido desarrollar su yo (self)), su propia subjetividad (42) (19). Por lo que su subjetividad tendrá que surgir por medio de otra persona.

Esta vulnerabilidad facilita el impacto de los factores precipitantes, que pueden ser identificados como procesos de separación, pérdida de figuras significativas, sea de forma real (muertes, separaciones, divorcios, abandonos de hogar) o simbólica. Así como la ruptura brusca de la homeostasis familiar por un problema crónico que provoca una situación estresante (adicción o enfermedad grave de un familiar). Y también la presencia de nuevas demandas en el entorno que se presentan de forma aguda o con escaso tiempo de elaboración y adaptación, pidiendo respuestas concretas en espacios cortos de tiempo.

La acción de estos factores precipitantes sobre la vulnerabilidad previa genera los síntomas que identifican a la codependencia.

Los factores de mantenimiento actúan sobre los factores precipitantes para que su acción haga que perdure la codependencia. Estos factores mantenedores son distorsiones cognitivas, tanto en su creencia de que su razonamiento es el que contiene mayor nivel de certeza como en la negación de sus necesidades básicas o en la confianza en una solución casi mágica de la adicción o enfermedad del otro.

De manera que el término codependencia hace referencia a que la dependencia es cosa de dos: un sumiso (dependiente manifiesto) y un controlador (dependiente larvado) que se necesitan mutuamente para la supervivencia emocional. Una caricatura adulta de la simbiosis original madre-hijo (11). 


\section{Abordaje}

Aunque la codependencia ha sido abordada con intervenciones breves de asesoramiento, con terapia familiar pasando por la terapia cognitiva, pocos Programas de Tratamiento en Europa hacen referencia a dicho concepto.

Aquí se propone la Psicoterapia Interpersonal que trataría de reconstruir la identidad dañada del codependiente a través del fomento de la autoestima, del reconocimiento de sus sentimientos, de potenciar sus habilidades relacionales, de favorecer su autoconcepto positivo y de su asertividad (43). De manera que el objetivo sería ir convirtiéndose en uno mismo, con actitudes, opciones y comportamientos libres.

Se ha señalado que la codependencia es una entidad diagnóstica curable (8). Pero sin un tratamiento adecuado puede convertirse en una adicción a sustancias químicas (19). Por lo que se incide en la necesidad ineludible de trabajar la independencia (44).

Este abordaje es una adaptación de la Psicoterapia Interpersonal Interpersonal (45) (46) que comenzó siendo utilizada en la depresión y más tarde en la distimia, en trastornos alimentarios, en el trastorno bipolar, en el trastorno boderline de la personalidad y en drogodependencias (47).

Tiene un enfoque pluralista, no doctrinario y empírico, y suele estar diseñada en un formato breve de unas 20 sesiones de periodicidad semanal de una hora de duración.

El rol del terapeuta es activo, de apoyo y poniéndose al lado del paciente. Y la relación terapéutica no es analizada como una manifestación de la transferencia.

La especifidad de la psicoterapia interpersonal son sus estrategias (48), no sus técnicas. Por lo que cabe la posibilidad de utilizar todas las técnicas que sirvan para reconstruir el propio pasado y abrir alternativas futuras y esperanzadoras suelen ser efectivas (49). De ahí su eclecticismo técnico.

\section{Estrategias}

Se organiza en tres fases:

En la fase primera se realiza un contrato o compromiso terapéutico explícito y se trata de relacionar la codependencia con el contexto interpersonal, revisando ordenadamente las relaciones interpersonales presentes y pasadas, ya que la codependencia es un estilo de vida y de relación.

Después se determinan los objetivos del tratamiento que pasarían por:

- Reconocer la existencia de un problema, ya que experimentan emociones negativas perturbadoras que les impiden dejar la relación insatisfactoria.

- Desprenderse emocionalmente de los problemas de los demás, porque no hay motivo para ser abandonado.

- Responder con acciones y no únicamente con preocupación, renunciando al rol de sufridor.

- Centrar la atención y la energía en su propia vida y no en otra relación.

- Asumir su responsabilidad no viviendo la situación con sentimiento de culpa (50), 
porque los codependientes suelen sentirse responsables últimos de la adicción o del problema del otro.

- Salir del ambiente familiar implica fortalecer las fronteras de la familia y abrir otros círculos: asociaciones, trabajo o voluntariado.

En la fase segunda se parte del supuesto básico de que la codependencia tiene que ver con problemas que se manifiestan en cuatro áreas-problemas:

- Duelo (porque pueden existir dificultades para la elaboración del duelo): se abordan las relaciones perdidas porque pueden existir dificultades para la elaboración de un duelo. Y se exploran los sentimientos asociados a la codependencia, tanto positivos como negativos y se analizan las ventajas y desventajas de esa relación.

- Disputas personales (porque pueden encontrarse situaciones interpersonales en las que se den expectativas no recíprocas): se abordan las relaciones alteradas porque pueden encontrarse situaciones interpersonales en las que se den expectativas no recíprocas. Se renegocian las diferencias de su relación con el adicto y con los demás miembros de la familia y de su red social.. Y se intenta recobrar la libertad de formar nuevos vínculos y realizar cambios activamente aunque no tengan éxito.

- Déficit interpersonales (porque puede haber una historia de empobrecimiento social): se relaciona la codependencia con los problemas de aislamiento social o de insatisfacción y se exploran las pautas repetitivas en las relaciones.

- Transiciones de rol (porque puede que haya cierto déficit para afrontar los cambios requeridos para el desempeño de otro rol): se abordan los roles perturbados porque puede que haya cierta dificultad para afrontar los cambios requeridos para otro rol. Y consiste en explorar las posibilidades de un nuevo rol más autónomo, en desarrollar nuevas habilidades para el desempeño del mismo y evaluar de forma realista lo que puede perderse.

Finalmente en la fase tercera se trata explícitamente de la finalización de la relación terapéutica, se reconoce que este final es un periodo de separación, despedida y duelo. Y se refuerzan los sentimientos de independencia del paciente y la capacidad de estar dispuesto a abandonar y a ser abandonado. 


\section{BIBLIOGRAFÍA}

1. Cocores, A.J. "Co.Adiction. A silent epidemic". Psychiatric Letter. Fair Oacks Hospital. 1987. 5, 2: 5-8.

2. Gómez Sanabria, A.; Gala León, F.J.; Lupiani, Giménez, M.; Bas Sarmiento, P.; Guillén Gestoso, C.; González, Rodriguez, R. "Aspectos cognitivos de la codependencia: Un estudio comparativo". V Encuentro Nacional sobre Drogodependencias y su Enfoque Comunitario. 1998. pp. 181-183.

3. Friel, J.C.; Friel, I.L. Adult children. The secrets of dysfunctional families. Deerfield Beach Florida. Health Communications, Inc. p. 157. 1988.

4. Jackson, J.K.; Kogan, K.L. "Personality disturbance in wives of alcoholics". Quaterly Journal of Studies on Alcohol. 1963. 24: 227-283.

5. Treadway, D. "Codependency: Disease, metaphor or fad?". Family Therapy Networker. 1990. 14, 1: 39-42.

6. Lawlor, E. M. "Creativity and change: The two-tiered creative arts therapy aproach to co-dependency treatment". The Arts in Psychotherapy. 1992. 19: 19-29.

7. Vacca Rodríguez, R. "Psicopatología de la co-adicción". Facetas. 1999. 4, 4: 73-112.

8. Gierymki, T.; Williams, T. "Codependency". Journal of Psychoactive Drugs. 1986. 18, 1: 7-13.

9. Subby, R. "Inside the chemically dependent marriage: Denial and manipulation". En J.Woititz, S. Wegscheider-Cruse y C. Whitfild (Eds.). Codependency: An emerging issue. Deerfield Beach. Florida. Health Communications, Inc. 1984.

10. Haaken, J. From Al-Anon to ACOA: "Codependence and the reconstruction of caregiving". Sings: Journal of Women in Culture and Society. 1993. 18, 2: 321-345.

11. May, D. Codependency: Powerloss Soullos. USA. Whales Tales Press and Paulist Press. 1994.

12. Cleveland, M. "Treatment of codependent women through the use of mental imagery". Alcoholism Treatment Quaterly. 1987. 4, 1: 27-40.

13. Gómez, Mª N.; Bolaños, C.; Rivero, M “¿Es la codependencia otro tipo de adicción en la mujer drogodependiente?". ler. Symposium Nacional sobre Adicción en la Mujer. Resúmenes de Ponencias y Comunicaciones. Madrid. Fundación Instituto Spiral. 2000. pp. 42-43.

14. Walters, J.W. "The codependent Cinderella who loves too much...fight back". Family Therapy Networker. 1990. 14, 4: 231-245.

15. Comunidad de Madrid. Problemas por el alcohol en la familia. Informe para la Unión Europea. Comunidad de Madrid Agencia Antidroga.. 1999. p. 47.

16. Anderson, S.C. "A critical analysis of the concept of codependency". Social Work. 1994. 39, 6: 677-684.

17. Llopis Llácer, J.J. "Determinantes de la adicción a la heroína en la mujer: la codependencia". Libro de Actas XXV Jornadas Nacionales de Socidrogalcohol. Tarragona. 1998. pp. 363-397.

18. Brown, S. Treating the alcoholic: A developmental model of recovery. New York. Wiley. 1985.

19. Jauregui, I. "Codependencia y literatura. La codependencia en la antigüedad clásica". Revista Española de Drogodependencias. 2000. 25, 4: 452-477.

20. Prest, L.A.; Storm, C. "The codependent relationships of compulsive eaters and drinkers: Drawing parallels". The Journal of Family Therapy. 1988. 16, 4: 339-350.

21. Wright, P.H.; Wright, K.D. "Codependency: Addictive love. Adjustive relating, or both?”. Contemporany Family Therapy. 1991. 13, 5: 47-63. 
22. Peele, S.; Brodsky, A. Love and addiction. New York. Signet. 1975.

23. Wegscheider-Cruse, S. "Codependency: The therapeutic void". En J.Woititz, S. WegscheiderCruse y C. Whitfild (Eds.). Codependency an emerging issue. Deerfield Beach. Florida. Health Communications, Inc. 1984.

24. Cermack, T.L. Diagnosis and treating codependence. Minneapolis. Jhonson Institute Books. 1986.25. Norwood, R. Women who love too. New York. Much. Basic. 1985.

26. Sirvent Ruíz, C. "Dependencias relacionales: codependencia, bidependencia y adicción afectiva". Ier. Symposium Nacional sobre Adicción en la Mujer. Resúmenes de Ponencias y Comunicaciones. Madrid. Fundación Instituto Spiral. pp. 27-30. 2000.

27. Lyles de Reagan, L. "La codependencia: Un problema familiar". www.christianrecovery.com/v/dox/coda.htm. 2001.

28. Coddou, A.; Chadwick, M. "Evolución del concepto de codependencia”. www:/mundomed.net/ revistas/era/numero7/61.html. 2001.

29. Beattie, M. "Características de la codependencia". www. christianrecovery.com/v/dox/coda.htm. 2001.

30. Kreuz, A. "La teoría sistémica y las drogodependencias: el rol de las familias en la prevención”. En J. Escámez (Ed.). Prevención de la Drogadicción. Valencia. Nau llibres. 1997.

31. Domínguez, M.D. "Problemática familiar en patología adictiva". XXII Jornadas Nacionales de Socidrogalcohol. 29-31 de Marzo 1995. Santiago de Compostela.

32. Bowlby, J. Attachment and loss. London. Hogarty Press. 1969.

33. Winnicot, D.W. Maturational processes and the facilitating environment. 1965. New York. International Univerity Press.

34. Klaus, M.H.; Kennel, J.H. Maternal infant bonding: the impact of early separation or loss on family development. Saint Louis. C.V. Mosby Company. 1976.

35. Wolff, P.H. "The causes, controls and organization of the behavior in the neonate". Psychological Issues Monograph. 1966. 17: 73-85.

36. Schaffer, R. Studies on interaction in infancy. London. Academic Press. 1977.

37. Brazelton, T.B.; Als, H. "Four early stages in the development of mother-infant interaction". Psychoanal. Study Child. 1979. 34, 349-369.

38. Levovici, S. Le bébé, la mère et le psychanalyste. Paris. Le Centurion. 1983.

39. Winnicot, D.W. The family and individual development. London. Tavistock Publications.1965.

40. Parker, G.; Tupling, G.; Brown, L.B. "A parental bonding instrument". British Journal of Med. Psych. 1982. 52: 1-10.

41. Cano de Escoriza, J. "El apego, factor clave en las relaciones interpersonales". Interpsiquis. www.psiquiatria.com/artículos/ psicología/2161/. 2001.

42. Lafond, C. "La mytho d'Echo ou l'imposible sujet". Revue FranÇaise de Psychoanalyse. 1991. 6: 1639-1644.

43. Goikoetxea, I.; Aizpiri, J.; Marcos, J.F. "Evolución de las esposas de los alcohólicos en tratamiento". Libro de Ponencias y Comunicaciones. XXI Jornadas Nacionales de Socidrogalcohol. 1994. pp. 387-410.

44. Hagan, T.A. et al. "Impediments to comprehensive treatment models for substance-dependent women: Treatment and research questions". Journal of Psychoactive Drugs. 1994. 26, 2: 263-171.

45. Klerman, G. L.; Weissman, M. M.; Rounsanville, B.J.; Chevron, E.S. Interpersonal psychotherapy with of depression (ITP). New York. Basic Boocks. 1984. 
ORIGINALES Y REVISIONES

46. Klerman, G. L.; Weissman, M. M. New applications of Interpersonal Psychotherapy. Washington. American Psychiatric Press. 1993.

47. Fernández, A.; Rodríguez, B.; Diéguez, Mª ; González, N.; Moralez, N. "La terapia interpersonal de la depresión y las psicoterapias en el fin del milenio". Revista de la Asociación Española de Neuropsiquiatría. 1997. XVII, 64: 627-648.

48. Schramm, E. Psicoterapia Interpersonal. Barcelona. Masson 1998..

49. Bannister, D. Issues and approaches in the psychological therapy. New York. Willey. 1975

50. Bononato, L. "Mujer y codependencia". Proyecto. 1996. 19: 9-11.

*Psicólogo.

Centro de Prevención del Alcoholismo y Tabaquismo. Ayuntamiento de Madrid.

Avda. Manzanares No 160, 28019-Madrid

Tlfno: 91.5606460

E-mail: ferdinan@correo.cop.es

Fecha de recepción: 7-2-02 